\title{
ELEMENTARY MODELS OF UNBOUNDED JACOBI MATRICES WITH A FEW BOUNDED GAPS IN THE ESSENTIAL SPECTRUM
}

\author{
Anne Boutet de Monvel, Jan Janas and Serguei Naboko
}

Abstract. This work contains a constructive example of a class of Jacobi operators with an arbitrary finite number of gaps in its unbounded essential spectrum. The construction of this class is based on elementary ideas of gluing finite-dimensional Jacobi matrices whose sizes grow to infinity. The precise analysis of the finite-dimensional pieces leads to a new "finite essential spectrum" besides the natural essential spectrum of two explicit infinite Jacobi matrices, determined by the above finite dimensional ones. This new finite essential spectrum is calculated explicitly. A connection to the ideas of the recent paper [12] is also given.

Mathematics subject classification (2010): Primary 47B36; Secondary 47A10, 47B25. Keywords and phrases: Jacobi matrix, essential spectrum, gaps, monodromy matrix.

\section{REFERENCES}

[1] N. I. AKHIEZER, The classical moment problem and some related questions in analysis, Translated by N. Kemmer. Hafner Publishing Co., New York, 1965.

[2] M. S. Birman And M. Z. Solomjak, Spectral theory of selfadjoint operators in Hilbert space, Mathematics and its Applications (Soviet Series), D. Reidel Publishing Co., Dordrecht, 1987.

[3] A. Boutet De Monvel, J. Janas, And S. NABoko, Unbounded Jacobi matrices with a few gaps in the essential spectrum. Constructive examples, Integral Equations Operator Theory, 69(2):151-170, 2011.

[4] P. A. CoJuhari, Discrete spectrum in the gaps for perturbations of periodic Jacobi matrices, Jour. Comput. Appl. Math., 225:374-386, 2009.

[5] J. DOMBROWS KI, Eigenvalues and spectral gaps related to periodic perturbations of Jacobi matrices, In Spectral methods for operators of mathematical physics, volume 154 of Oper. Theory Adv. Appl., pages 91-100. Birkhäuser, Basel, 2004.

[6] J. Dombrowski, J. Janas, M. Moszyński, And S. Pedersen, Spectral gaps resulting from periodic perturbations of a class of Jacobi operators, Constr. Approx., 20(4):585-601, 2004.

[7] V. Georgescu, On spectral analysis of quantum field Hamiltonians, J. Func. Anal., 245(1):89-143, 2007.

[8] I. M. Glazman, Direct methods of qualitative spectral analysis of singular differential operators, Translated from the Russian by the IPST staff. Israel Program for Scientific Translations, Jerusalem, $1965,1966$.

[9] J. JANAS AND M. MosZYŃSKI, Spectral properties of Jacobi matrices by asymptotic analysis, J. Approx. Theory, 120(2):309-336, 2003.

[10] J. JANAS, S. NABOKo, AND G. Stolz, Decay bounds on eigenfunctions and the singular spectrum of unbounded Jacobi matrices, Int. Math. Res. Notices, 4:736-764, 2009.

[11] T. Kato, Perturbation theory for linear operators, Classics in Mathematics. Springer-Verlag, Berlin, 1995. Reprint of the 1980 edition.

[12] Y. Last And B. Simon, The essential spectrum of Schrödinger, Jacobi, and CMV operators, J. Anal. Math., 98:183-220, 2006.

[13] M. Moszyński, Spectral properties of some Jacobi matrices with double weights, J. Math. Anal. Appl., 280(2):400-412, 2003. 
[14] V. S. Rabinovich, S. Roch And B. Silbermann, Limit Operators and Their Applications in Operator Theory, Birkhäuser, 2004.

[15] G. TeschL, Jacobi operators and completely integrable nonlinear lattices, volume 72 of Mathematical Surveys and Monographs, American Mathematical Society, Providence, RI, 2000. 